\title{
Iminoglycinuria and hyperglycinuria are discrete human phenotypes resulting from complex mutations in proline and glycine transporters
}

\author{
Stefan Bröer, ${ }^{1}$ Charles G. Bailey,, ${ }^{2}$ Sonja Kowalczuk, ${ }^{1}$ Cynthia Ng, ${ }^{2}$ Jessica M. Vanslambrouck, ${ }^{2}$ \\ Helen Rodgers, ${ }^{3}$ Christiane Auray-Blais, ${ }^{4}$ Juleen A. Cavanaugh, ${ }^{3}$ \\ Angelika Bröer, ${ }^{1}$ and John E.J. Rasko ${ }^{2,5}$

\begin{abstract}
1School of Biochemistry and Molecular Biology, Australian National University, Canberra, Australian Capital Territory, Australia. ${ }^{2}$ Centenary Institute, Camperdown, New South Wales, Australia. ${ }^{3}$ Medical Genetics Research Unit, Australian National University Medical School, Research School of Biological Sciences, Australian National University, Canberra, Australian Capital Territory, Australia. ${ }^{4}$ Service of Genetics, Department of Pediatrics, Université de Sherbrooke, Sherbrooke, Quebec, Canada. ${ }^{5}$ Cell and Molecular Therapies, Sydney Cancer Centre, Royal Prince Alfred Hospital, Camperdown, New South Wales, Australia.
\end{abstract}

\begin{abstract}
Iminoglycinuria (IG) is an autosomal recessive abnormality of renal transport of glycine and the imino acids proline and hydroxyproline, but the specific genetic defect(s) have not been determined. Similarly, although the related disorder hyperglycinuria (HG) without iminoaciduria has been attributed to heterozygosity of a putative defective glycine, proline, and hydroxyproline transporter, confirming the underlying genetic defect(s) has been difficult. Here we applied a candidate gene sequencing approach in 7 families first identified through newborn IG screening programs. Both inheritance and functional studies identified the gene encoding the proton amino acid transporter SLC36A2 (PAT2) as the major gene responsible for IG in these families, and its inheritance was consistent with a classical semidominant pattern in which 2 inherited nonfunctional alleles conferred the IG phenotype, while 1 nonfunctional allele was sufficient to confer the HG phenotype. Mutations in SLC36A2 that retained residual transport activity resulted in the IG phenotype when combined with mutations in the gene encoding the imino acid transporter SLC6A20 (IMINO). Additional mutations were identified in the genes encoding the putative glycine transporter SLC6A18 (XT2) and the neutral amino acid transporter SLC6A19 ( $\left.B^{0} \mathrm{AT} 1\right)$ in families with either IG or HG, suggesting that mutations in the genes encoding these transporters may also contribute to these phenotypes. In summary, although recognized as apparently simple Mendelian disorders, IG and HG exhibit complex molecular explanations depending on a major gene and accompanying modifier genes.
\end{abstract}

\section{Introduction}

Many metabolic diseases, such as Hartnup disorder (1) and phenylketonuria (2), have been assumed to fit a classical Mendelian autosomal model of inheritance. In classical Mendelian diseases such as hemochromatosis (3), reduced penetrance is often invoked to explain the failure to develop disease despite inheritance of 2 abnormal alleles. Iminoglycinuria (IG; OMIM 242600) in some families also exhibits a more complex inheritance pattern, including reduced penetrance (4). Both parents from the family in which IG was first identified exhibited a normal urinary phenotype (5), whereas later studies described families in which the parents exhibited hyperglycinuria (HG; OMIM 138500) without iminoaciduria (6-8). Consequently, IG was thought to arise when 2 defective alleles of a common transporter for glycine, proline, and hydroxyproline are present (9), whereas heterozygosity of the putative defective transporter would manifest as HG. Observed exceptions to this pattern led to speculation regarding "incompletely recessive" forms or the involvement of alleles with different molecular

Nonstandard abbreviations used: cRNA, complementary RNA; HG, hyperglycinuria; IG, iminoglycinuria.

Conflict of interest: The authors have declared that no conflict of interest exists. Citation for this article: J. Clin. Invest. doi:10.1172/JCI36625 outcomes $(4,10)$. However, in the absence of an explanation of its molecular pathogenesis, the genetic complexity of IG has remained obscure since its first description more than 50 years ago.

Although generally classified as benign inborn errors of amino acid transport (11), IG and HG have been associated with hypertension, glycosuria (12), nephrolithiasis (OMIM 138500; known as IG type II) $(13,14)$, mental retardation (15), atypical gyrate atrophy (16), deafness $(8)$, and blindness $(17,18)$. In the absence of controlled prospective studies of IG and HG cohorts, some of these associations may represent ascertainment bias. Based on detailed biochemical studies in 7 families, Lasley and Scriver (19) suggested that 3 genes account for imino acid and glycine reabsorption in the kidney proximal tubule, namely a common transporter for both types of amino acids and a specific transporter each for glycine and imino acids. This model of 1 common and 2 specific transporters is well supported by transport studies in intact tubules, brush-border membrane vesicles, and cell lines from a wide variety of organisms (20).

In the absence of sufficient family material to undertake traditional linkage analysis, we adopted a candidate gene sequencing approach targeting recently identified epithelial proline and glycine transporters to dissect the molecular pathogenesis of IG and HG. Comparison of earlier physiological data with the properties of cloned proline and glycine transporters suggests that all major 

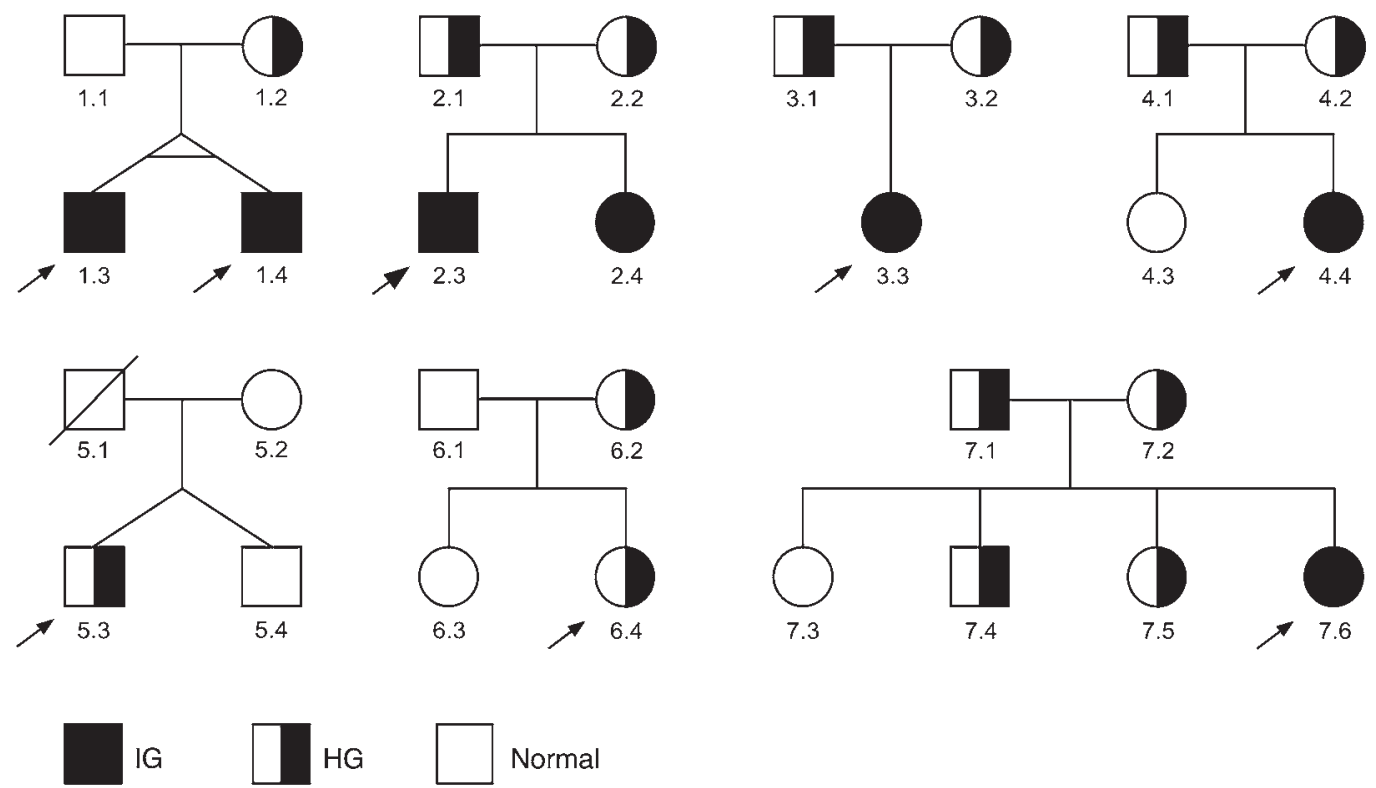

Figure 1

Pedigrees with HG or IG. Filled symbols represent individuals with IG; half-filled symbols, those with HG. Open symbols represent normal family members. An arrow indicates the index case. Pedigrees 1-3 are French-Canadian; pedigrees 4-7 are Australian; pedigree 7 is of Coptic-Egyptian descent. The urine of subject 6.1 was not tested, and subject 5.1 was deceased.

mediators of proline and glycine transport have been identified at the molecular level (20) in recent years. Two common transporters have been cloned that cotransport proline, hydroxyproline, and glycine together with protons, namely SLC36A1 (PAT1) and SLC36A2 (PAT2) (21). A major feature distinguishing these transporters is their substrate affinity: SLC36A1 is a low-affinity transporter, whereas SLC36A2 is a high-affinity transporter. SLC36A1 has been characterized as the intestinal imino acid and glycine transporter and suggested as a candidate for IG $(22,23)$. More recently, 2 groups including ours identified SLC6A20 (IMINO) as a specific imino acid transporter $(24,25)$. Studies in the SLC6A18 nullizygous mouse suggest that this gene encodes a high-affinity renal epithelial transporter specific for glycine (26); however, attempts to functionally express SLC6A18 have not been successful $(27,28)$. Physiological and genetic data also demonstrate that the general neutral amino acid transporter SLC6A19, which is mutated in Hartnup disease (28-30), contributes significantly to imino acid and glycine transport in kidney and intestine $(31,32)$. In this report, we describe 2 novel SLC $36 A 2$ mutations, one allele of which apparently acts in a semidominant manner, while a second allele appears to be acting recessively. Our results strongly suggest that the SLC36A2 mutations together with polymorphisms in the modifiers SLC6A20, SLC6A18, and SLC6A19 constitute the genetic basis for these intriguing human phenotypes.

\section{Results}

Seven families, including French-Canadians (pedigrees 1-3) and Australians (pedigrees 4-7), each containing an index case initially identified from newborn urinary screening programs for IG $(33,34)$, were recruited for this study (Figure 1). Most probands who had IG diagnosed as infants (subjects 1.3, 1.4, 2.3, 2.4, 3.3, 4.4, and 7.6) were reconfirmed in this study, whereas subjects 5.3 and 6.4 persisted with only HG. The term HG included any indi- vidual exhibiting elevated urinary glycine levels on at least one occasion. All urinary amino acid quantitations from the 7 pedigrees are shown in Supplemental Table 1 (supplemental material available online with this article; doi:10.1172/JCI36625DS1). The phenotypic distinction between HG and IG was unambiguous: excess urinary proline and hydroxyproline (always accompanied by excess glycine) indicated IG, whereas isolated excess urinary glycine indicated HG. The variability in phenotype observed in these pedigrees, particularly between parents, suggested the possibility that genetic heterogeneity was a contributing factor.

Genomic DNA samples from all 7 IG and HG families were sequenced to identify mutations in known imino acid and glycine transporter candidates SLC36A1, SLC36A2, SLC6A20, SLC6A18, and SLC6A19, including coding regions and exon-intron boundaries. Mutations causing nonsense, missense, and splice site changes and polymorphisms of uncertain significance are summarized in Table 1. Mutations that cosegregated with the IG and HG phenotypes were detected in the genes encoding SLC36A2 (the common glycine and imino acid transporter), SLC6A20 (the specific imino acid transporter), and SLC6A18 (the specific glycine transporter). Despite biochemical evidence for the candidacy of SLC36A1, no mutations segregating with the urinary phenotypes were identified in that gene.

The main contribution to IG and HG arises from defects in the imino acid and glycine transporter SLC36A2 (Table 1), thereby proving the assumption that the common transporter is the major gene contributing to both IG and HG. A $260 \mathrm{G} \rightarrow$ T mutation resulting in the substitution of a highly conserved glycine residue by valine $(\mathrm{G} 87 \mathrm{~V})$ in the second transmembrane helix of SLC36A2 (Figure $2 \mathrm{~A}$ and Figure $3 \mathrm{~A}$ ) was identified in 5 pedigrees. In pedigree 7 (of Coptic-Egyptian descent), a splice donor site mutation in the first intron of SLC36A2, IVS1+1G $\rightarrow$ A, was identified that was predicted $(35,36)$ to impair normal splicing. In addition, a 


\section{Table 1}

Mutations and polymorphisms identified in SLC36A2 and SLC6 amino acid transporter (II) family members that contribute to HG and IG

\begin{tabular}{|c|c|c|c|c|c|c|c|c|c|}
\hline \multicolumn{2}{|l|}{ Gene } & \multicolumn{2}{|c|}{ SLC36A2 } & SLC6A20 & \multicolumn{4}{|c|}{ SLC6A18 } & \multirow{3}{*}{$\begin{array}{c}\text { SLC6A19 } \\
5 p 13.33 \\
\text { IVS7-4G } \rightarrow \text { A }\end{array}$} \\
\hline Locus & & & 33.1 & $3 p 21.3$ & & $5 p 1$ & 3.33 & & \\
\hline Allele & & $260 \mathrm{G} \rightarrow \mathrm{T}$ & IVS1+1G $\rightarrow A$ & $596 C \rightarrow T$ & $235 \mathrm{G} \rightarrow \mathrm{A}$ & $957 \mathrm{C} \rightarrow \mathrm{G}$ & $1433 \mathrm{~T} \rightarrow \mathrm{C}$ & $1486 \mathrm{G} \rightarrow \mathrm{A}$ & \\
\hline Mutation/S & NP & G87V & IVS1+1G $\rightarrow A$ & T199M & G79S & Y319X & L478P & G496R & IVS7-4G $\rightarrow A$ \\
\hline Frequency & & 0.012 & 0.004 & 0.075 & ND & 0.441 & 0.386 & 0.003 & 0.22 \\
\hline RefSNP ID & & & & rs17279437 & & rs7447815 & rs4073918 & & rs35329108 \\
\hline Subject & Phenotype & & & & & & & & \\
\hline 1.1 & NM & $+/-$ & $+/+$ & $+/+$ & $+/+$ & $+/+$ & $+/-$ & $+/+$ & $-1-$ \\
\hline 1.2 & $H G$ & $+/-$ & t/t & $+1-$ & $+/+$ & $+1-$ & t/t & $+/+$ & $+/-$ \\
\hline 1.3 & IG & $-1-$ & $+/+$ & $+/-$ & $+/+$ & $+/-$ & $+/-$ & $+/+$ & $+/-$ \\
\hline 1.4 & IG & $-1-$ & $+/+$ & $+/-$ & $+/+$ & $+/-$ & $+1-$ & $+/+$ & $+1-$ \\
\hline 2.1 & $H G$ & $-1-$ & t/t & $+/+$ & t/t & $+/+$ & $-1-$ & $+/+$ & $+/+$ \\
\hline 2.2 & $H G$ & $+/-$ & $+/+$ & $+/-$ & $+/+$ & $+/-$ & $+/-$ & $+/+$ & $t / t$ \\
\hline 2.3 & $\mathrm{IG}$ & $-1-$ & $+/+$ & $+/-$ & $+/+$ & $+/+$ & $-1-$ & $+/+$ & $+/+$ \\
\hline 2.4 & IG & $-1-$ & $+/+$ & $+/-$ & $+/+$ & $+/+$ & $-1-$ & $+/+$ & $+/ t$ \\
\hline 3.1 & $H G$ & $+/-$ & $+/+$ & $+/-$ & $+/+$ & $+/+$ & $+/-$ & $+/+$ & $+/+$ \\
\hline 3.2 & $\mathrm{HG}$ & $+1-$ & $+/+$ & $+/+$ & $+/+$ & $+/-A$ & $+/-A$ & $+/+$ & $+/+$ \\
\hline 3.3 & $I G$ & $-/-$ & $+/+$ & $+/-$ & $+/+$ & $+/+$ & $-1-$ & $+/+$ & $+/+$ \\
\hline 4.1 & $\mathrm{HG}$ & $+/-$ & $+/+$ & $+/+$ & $+/+$ & $+/+$ & $+/+$ & $+/+$ & $+/-$ \\
\hline 4.2 & $\mathrm{HG}$ & $+1-$ & $+/+$ & $+1-$ & $+/+$ & $+1-$ & $+/+$ & $+/+$ & $+/-$ \\
\hline 4.3 & NM & $+/+$ & $+/+$ & $+1-$ & $+/+$ & $+/+$ & $+/+$ & $+/+$ & $+1-$ \\
\hline 4.4 & $I G$ & $-1-$ & $+/+$ & $+/+$ & $+/+$ & $+/+$ & $+/+$ & $+/+$ & $-1-$ \\
\hline 5.1 & DEC & $\mathrm{N} / \mathrm{A}$ & $\mathrm{N} / \mathrm{A}$ & $\mathrm{N} / \mathrm{A}$ & $\mathrm{N} / \mathrm{A}$ & $\mathrm{N} / \mathrm{A}$ & $\mathrm{N} / \mathrm{A}$ & $\mathrm{N} / \mathrm{A}$ & $\mathrm{N} / \mathrm{A}$ \\
\hline 5.2 & NM & $+/+$ & $+/+$ & $+/+$ & $+/+$ & $-/-$ & $+/+$ & $+/+$ & $+/+$ \\
\hline 5.3 & $H G$ & $+/-$ & $+/+$ & $+/-$ & $+/-$ & $+/-$ & $+/+$ & $+/+$ & $+/-$ \\
\hline 5.4 & NM & $+/+$ & $+/+$ & $+/+$ & $+/+$ & $+1-$ & $+/+$ & $+/+$ & $+/+$ \\
\hline 6.1 & N/A & $\mathrm{N} / \mathrm{A}$ & $\mathrm{N} / \mathrm{A}$ & $\mathrm{N} / \mathrm{A}$ & $\mathrm{N} / \mathrm{A}$ & $\mathrm{N} / \mathrm{A}$ & $\mathrm{N} / \mathrm{A}$ & $\mathrm{N} / \mathrm{A}$ & $\mathrm{N} / \mathrm{A}$ \\
\hline 6.2 & $\mathrm{HG}$ & $+/+$ & $+/+$ & $-1-$ & $+/+$ & $+/-$ & $+/+$ & $+/+$ & $+/-$ \\
\hline 6.3 & NM & $+/+$ & $+/+$ & $+/+$ & $+/+$ & $+1-$ & $+/+$ & $+/+$ & $+/+$ \\
\hline 6.4 & $H G$ & $+/+$ & $+/+$ & $+/-$ & $+/+$ & $+/-$ & $+/-$ & $+/+$ & $+/-$ \\
\hline 7.1 & $H G$ & $+/+$ & $+1-$ & $+/+$ & $+/+$ & $+/+$ & $+/+$ & $+/-$ & $-1-$ \\
\hline 7.2 & $\mathrm{HG}$ & $+/+$ & $+/-$ & $+/+$ & $+/+$ & $+/-$ & $+/+$ & $+/+$ & $+1-$ \\
\hline 7.3 & NM & $+/+$ & $+/+$ & $+/+$ & $+/+$ & $+/+$ & $+/+$ & $+/+$ & $-1-$ \\
\hline 7.4 & $\mathrm{HG}$ & $+/+$ & $+/-$ & $+/+$ & $+/+$ & $+/+$ & $+/+$ & $+/-$ & $-1-$ \\
\hline 7.5 & $\mathrm{HG}$ & $+/+$ & $+/-$ & $+/+$ & $+/+$ & $+/-$ & $+/+$ & $+1-$ & $+/-$ \\
\hline 7.6 & $I G$ & $+/+$ & $-/-$ & $+/+$ & $+/+$ & $+/-$ & $+/+$ & $+/-$ & $+/-$ \\
\hline
\end{tabular}

Each subject is listed with phenotype: IG, HG, and normal (NM); and with genotype from the 4 candidate genes SLC36A2 (RefSeq NM_181776.1), SLC6A20 (NM_022405.2), SLC6A18 (NM_182632.1), and SLC6A19 (NM_001003841). N/A, not available; ND, not determined; DEC, deceased. AMutations on the same allele. All SLC6A18 mutations/SNPs were compound heterozygous unless otherwise indicated. RefSNPs are shown with identifiers and frequencies where known; frequencies of novel mutations were calculated in a panel of healthy individuals.

common SNP, 596C $\rightarrow$ T (frequency, 0.075; Table 1), in SLC6A20 that causes a substitution of threonine by methionine (T199M) in the fifth transmembrane region (Figure $2 \mathrm{~B}$ and Figure $3 \mathrm{~B}$ ) was identified in 6 pedigrees. In families 1-3, IG was only observed when homozygous SLC36A2 G87V was combined with SLC6A20 T199M. By contrast, homozygosity of the IVS1 $+1 \mathrm{G} \rightarrow$ A mutation in SLC36A2 appeared to be sufficient to cause IG without additional polymorphisms in SLC6A20.

To explain this difference, we analyzed the functional consequences of these mutations. In in vitro models, all mutations identified in SLC36A2 and SLC6A20 were found to functionally impair or abrogate transport (Figures 4-6). SLC36A2 was partially inactivated by the G87V mutation, displaying about $50 \%$ of the transport activity of the WT for both proline and glycine at physiological concentrations $(100 \mu \mathrm{M})($ Figure $4 \mathrm{~A})$. The reduced transport activity of SLC36A2 $\mathrm{G} 87 \mathrm{~V}$ was due to shift of the substrate concentration dependence (Figure 4, B and C) resulting in an increased $K_{m}$ value, which is visible as a shift of the curve to the right. In the case of proline, the $K_{m}$ value increased from $0.14 \pm 0.01 \mathrm{mM}$ to $0.39 \pm 0.02 \mathrm{mM}$ and in the case of glycine, from $0.49 \pm 0.07 \mathrm{mM}$ to $2.35 \pm 0.11 \mathrm{mM}$. The maximum transport velocity (Figure 4, B and C) and surface expression (Figure 4, D-F) were preserved in the G87V mutant.

The consequence of the IVS1+1G $\rightarrow$ A splice donor site mutation on SLC36A2 function was investigated using a minigene approach. The data in Figure 5 confirm that this mutation resulted in the use of an alternative splice site $70 \mathrm{bp}$ downstream of the WT donor splice site, which in turn led to truncation of the protein, thereby abrogating its expression (Supplemental Figure 1). SLC6A20 was almost completely inactivated by the SNP T199M (Figure 4A and Figure 6) due to a reduction of the maximum velocity from $282 \pm 6$ $\mathrm{pmol} / 10 \mathrm{~min}$ to $35 \pm 3 \mathrm{pmol} / 10 \mathrm{~min}$ per oocyte, which affected substrate dependence (Figure 6A), $\mathrm{Na}^{+}$dependence (Figure 6B), and $\mathrm{Cl}^{-}$dependence (Figure 6C). Similar to the SLC36A2 G87V mutant, surface expression was not altered (Figure 6, D-F).

A further level of complexity was observed in the putative glycine transporter SLC6A18. A number of SNPs were identified in all 
A

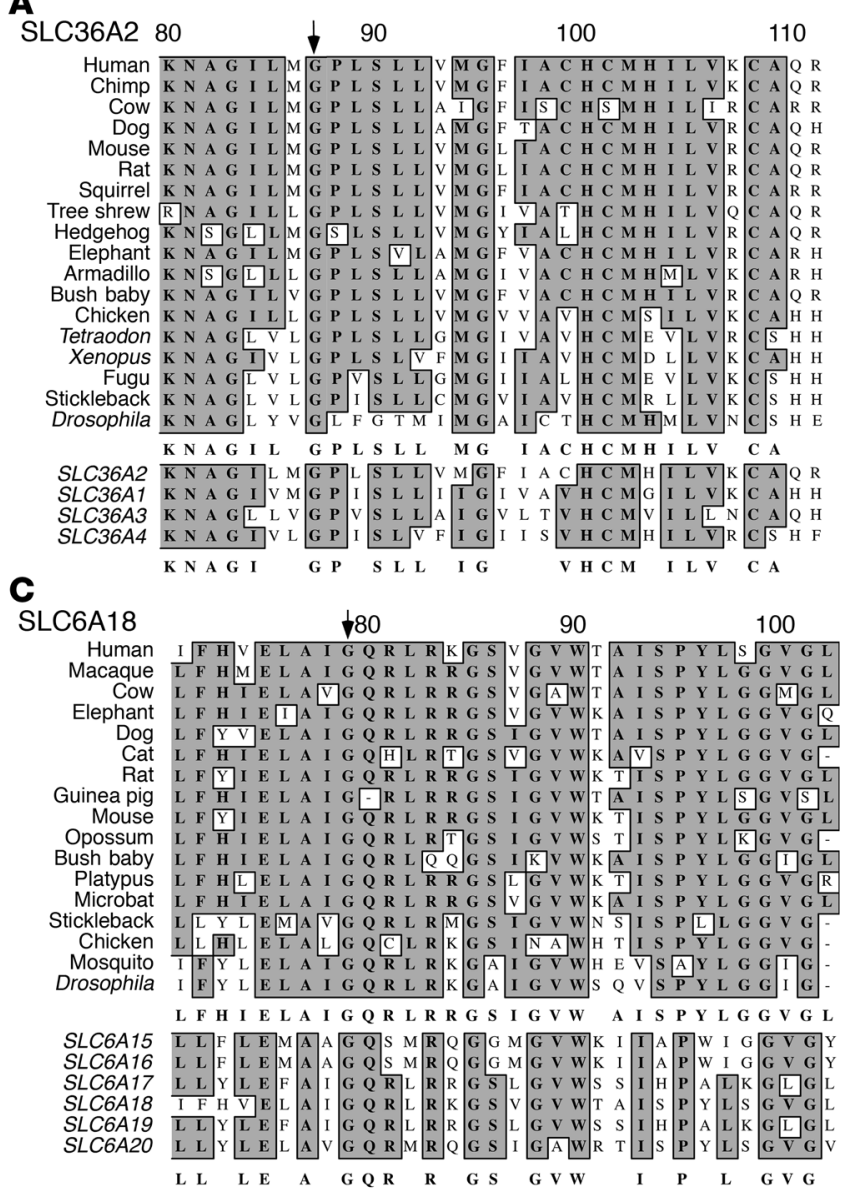

B

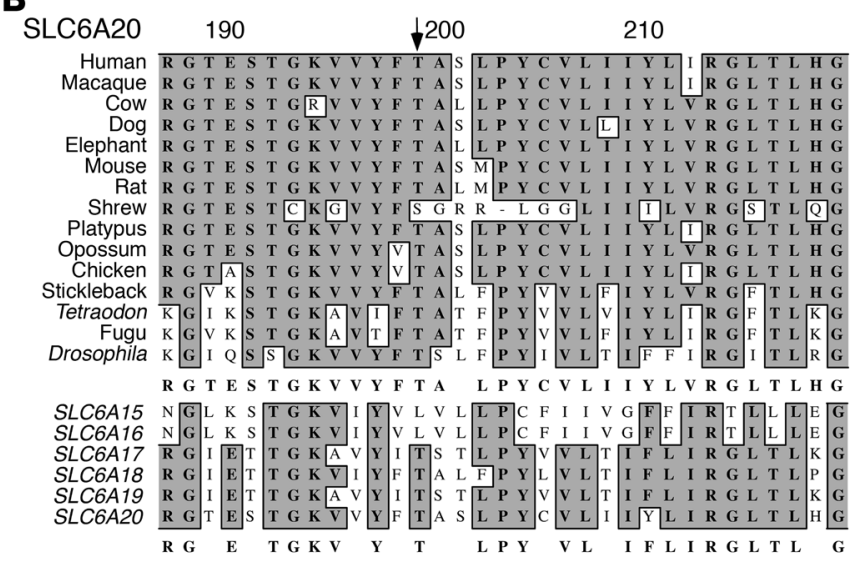

D

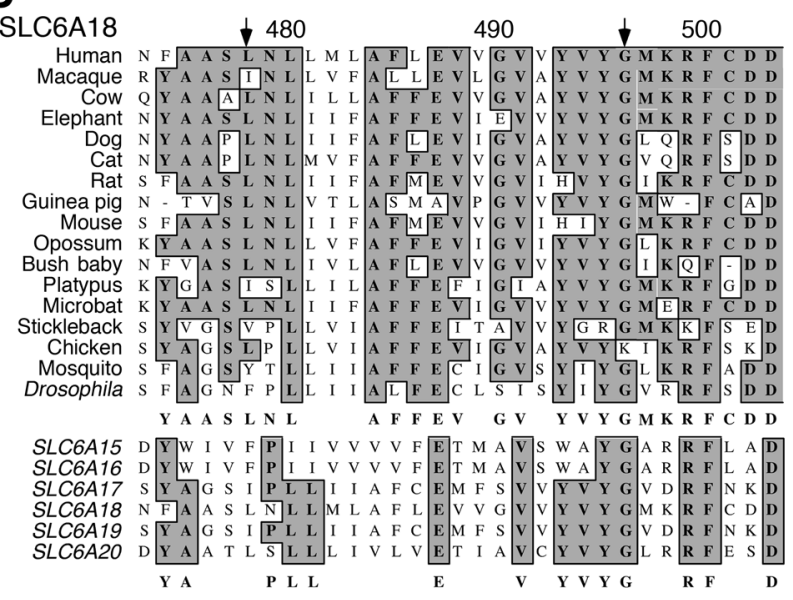

Figure 2

Mutations identified in IG and HG candidate genes SLC36A2, SLC6A20, and SLC6A18 encode highly conserved amino acid residues in transmembrane regions. Multiple amino acid sequence alignments were performed using ClustalW. Human RefSeq protein SLC36A2 (NP_861441.1) was aligned with SLC36A1 (NP_510968.2), SLC36A3 (NP_861439.2), and SLC36A4 (NP_689526.2). SLC6A20 (NP_064593.1) and SLC6A18 (NP_872438.1) were aligned with members of the SLC6 amino acid transporter (II) family (39): SLC6A15 (NP_877499.1); SLC6A16 (NP_054756.2); SLC6A17 (NP_001010898.1); and SLC6A19 (NP_001003841.1). Orthologs from other species were sourced from Ensembl release 47. Gray shading indicates residues that are conserved in the majority of sequences. Mutations identified in this study are indicated with arrows: (A) SLC36A2 G87V; mutation is in the second transmembrane helix; (B) SLC6A20 T199M; mutation is in the fifth transmembrane helix; (C) SLC6A18 G79S; mutation is in the second transmembrane helix; and (D) SLC6A18 L478P and G496R, both located in the tenth transmembrane helix.

families, but not in all individuals with IG or HG. These included known SNPs 957C $\rightarrow \mathrm{G}(\mathrm{Y} 319 \mathrm{X})$ and $1433 \mathrm{~T} \rightarrow \mathrm{C}(\mathrm{L} 478 \mathrm{P})(37)$ and novel mutations $235 \mathrm{G} \rightarrow \mathrm{A}(\mathrm{G} 79 \mathrm{~S})$ and $1486 \mathrm{G} \rightarrow \mathrm{A}(\mathrm{G} 496 \mathrm{R})$. All missense mutations/SNPs occurred in transmembrane domain residues that are highly conserved between SLC6A18 orthologs and within the amino acid transporter branch (II) of the SLC6 family $(38,39)$ (Figure 2, C and D, and Figure 3C). Although SLC6A18 could not be functionally expressed in heterologous systems (27, 28), surface expression of mutants G79S and G496R in oocytes was abrogated (Supplemental Figure 2, B and E), truncated protein Y319X (Supplemental Figure 2C) was reduced, and the L478P protein was normal (Supplemental Figure 2D).

The partial inactivation of SLC36A2 by the G87V mutation explained why IG was only observed when a homozygous mutation was accompanied by haploinsufficiency of SLC6A20 in pedigrees 1-3 (Table 1). In the presence of normal gene sequences for SLC6A20, SLC36A1, and SLC6A18, the homozygous SLC36A2 G87V mutation detected in subject 4.4 did not explain the iminoaciduria and marked
HG. Consequently, we identified a known polymorphism in SLC6A19 within the splice acceptor site of intron 7 , IVS7-4G $\rightarrow$ A, that cosegregated with IG (Table 1 ) and was predicted by information theorybased analysis $(35,36)$ to affect splicing and branch site selection.

Altered function in imino acid and glycine transporters revealed by the genotype and phenotype of subjects with HG and IG is consistent with their expression in the nephron. SLC36A2, the common high-capacity proline and glycine transporter, was localized within the S1 segment of the most proximal part of the convoluted tubule adjacent to the glomerulus (Figure 7A). In contrast, the expression of SLC6A20 was restricted to S2 segments in the renal cortex and S3 straight tubules traversing into the medulla (Figure 7B), thus establishing a mutually exclusive renal tubular distribution of SLC36A2 and SLC6A20 (Figure 7C).

\section{Discussion}

In order to explain the urinary phenotypes of IG and HG, we have identified mutations in several amino acid transporters that are 
$\mathbf{A}$ SLC36A2

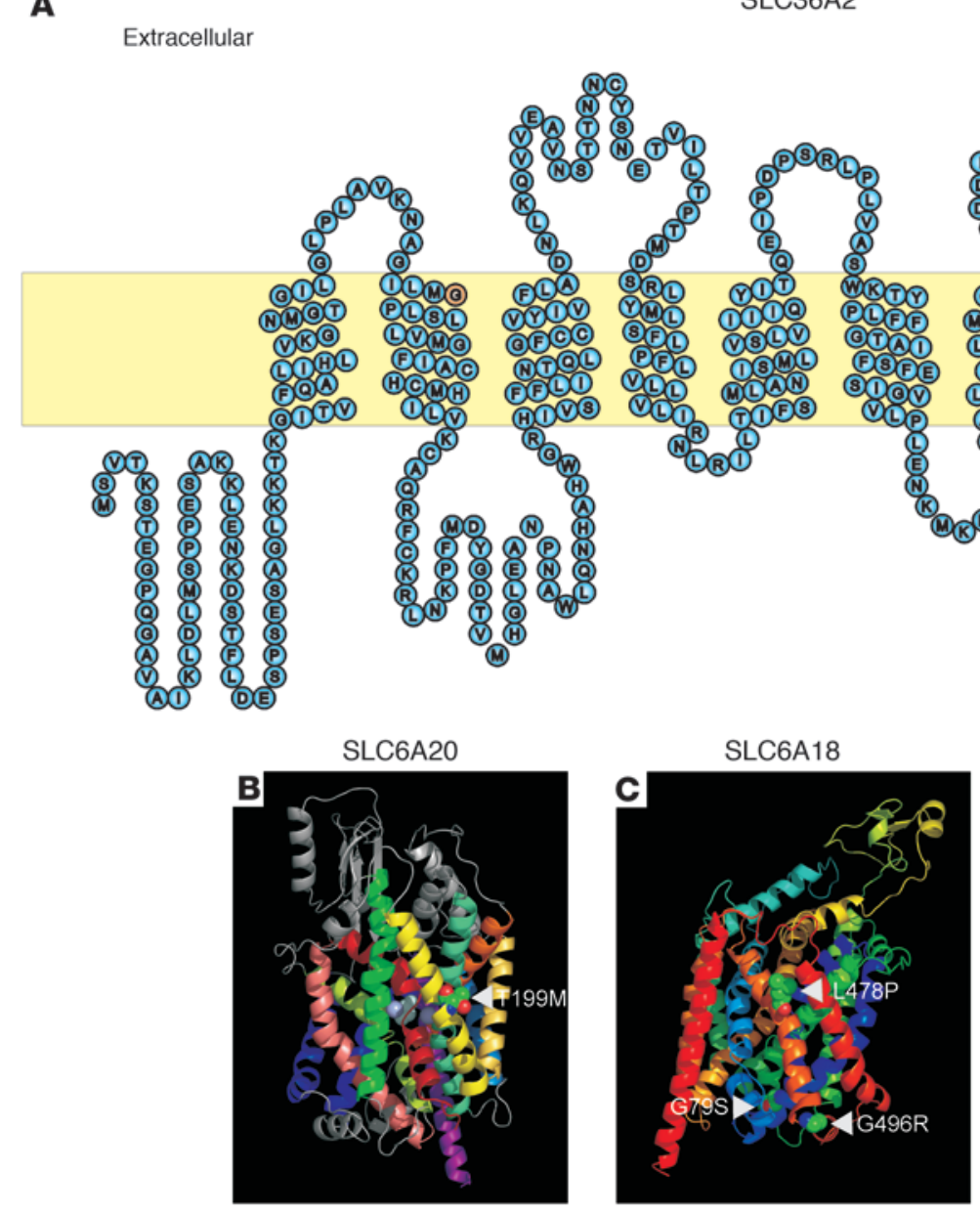

\section{Figure 3}

Location of IG-associated mutations in SLC36A2, SLC6A18, and SLC6A20. A topology model suggests location of residue $\mathrm{G} 87$ at the beginning of helix 2 (orange, A). Homology models of SLC6A20 (B) and SLC6A18 (C) were generated according to the structure of LeuT (51). Affected residues are indicated and shown in van der Waals projection. Both G79 and G496 are located at or close to critical helical turns, whereas L478 is located in the center of helix 10. known to be involved in the reabsorption of proline and glycine in the kidney (20). The major gene involved in IG and HG is the common imino acid and glycine transporter SLC36A2. We identified 2 mutant alleles, one of which completely (IVS1+1G $\rightarrow$ A) and the other partially (G87V) abolished its activity. The SNP T199M in SLC6A20 was observed in 6 of the 7 independent pedigrees and almost completely inactivated SLC6A20 in an in vitro model. In the putative glycine transporter SLC6A18, mutations G79S and G496R abolished surface expression, while the SNP Y319X introduced a stop codon. We have excluded the previously suggested candidate amino acid transporter SLC36A1 (22) and instead demonstrated a central causative role for SLC36A2 as well as SLC6A20. The contributions of SLC6A18 and SLC6A19 are discussed below.

The complex genetics of IG and HG is illuminated by examination of specific pedigrees presented in Table 1 . Inheritance of 2 SLC36A2-null alleles (IVS1+1G $\rightarrow$ A) in individual 7.6 was the only example of the 7 cases of IG explained by a simple autosomal recessive model. In the remaining 6 cases of IG, reduced activity of SLC36A2 due to homozygous G87V mutations was present, but additional haploinsufficiency of the proline transporter SLC6A20 due to the T199M allele was observed in subjects 1.3, 1.4, 2.3, 2.4, and 3.3. The combination of single alleles of SLC36A2 G87V and SLC6A20 T199M (individuals 1.2, 2.2, and 3.1) was not sufficient to cause IG. Thus, the genetic model consistent with the observed pattern of inheritance associated with the SLC36A2 G87V muta- tion is classical semidominant inheritance, albeit with additional contributions from modifier loci. Heterozygosity of the null mutation SLC36A2 IVS1+1G $\rightarrow$ A resulted in $\mathrm{HG}$, as seen in 7.1, 7.2, 7.4, and 7.5. Partial inactivation of SLC36A2 due to the G87V mutation alone also caused HG in all but 1 (subject 1.1) of the 8 heterozygotes. In 6 of the 7 SLC36A2 G87V heterozygotes with HG (subjects $1.2,2.2,3.1,3.2,4.2$, and 5.3), additional inactivating mutations in SLC6A18 (G79S, Y319X) were found.

Neonates frequently exhibit "physiological" IG, which disappears after "maturation" of kidney function $(11,40,41)$. This developmental IG has been attributed to a delay in the expression of transporters (42), which we now propose to involve reduced function of the common transporter SLC36A2 combined with the specific imino acid transporter SLC6A20 and the putative glycine transporter SLC6A18. In the pedigrees we studied, most cases of IG detected at birth persisted to adulthood, but in 2 subjects (nos. 5.3 and 6.4), it resolved to HG or normality. The genotypes in subjects 5.3 and 6.4 would lead to partial loss of proline and glycine transport capacity that would result in IG at birth, but maturation of the remaining intact transporters would compensate in adulthood (Table 1).

Homozygosity of the SLC6A20 T199M SNP alone did not result in prolinuria or IG (see subject 6.2), suggesting that the bulk of proline reabsorption is carried out by SLC36A2, the major gene involved in IG. In contrast to the impaired glycine transport and 

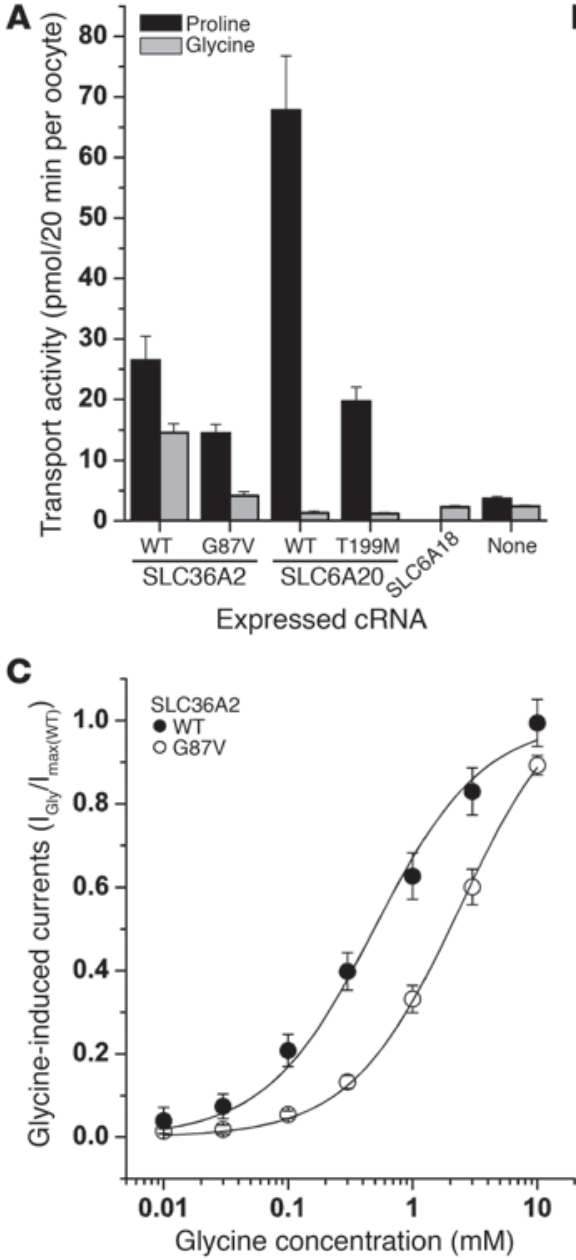
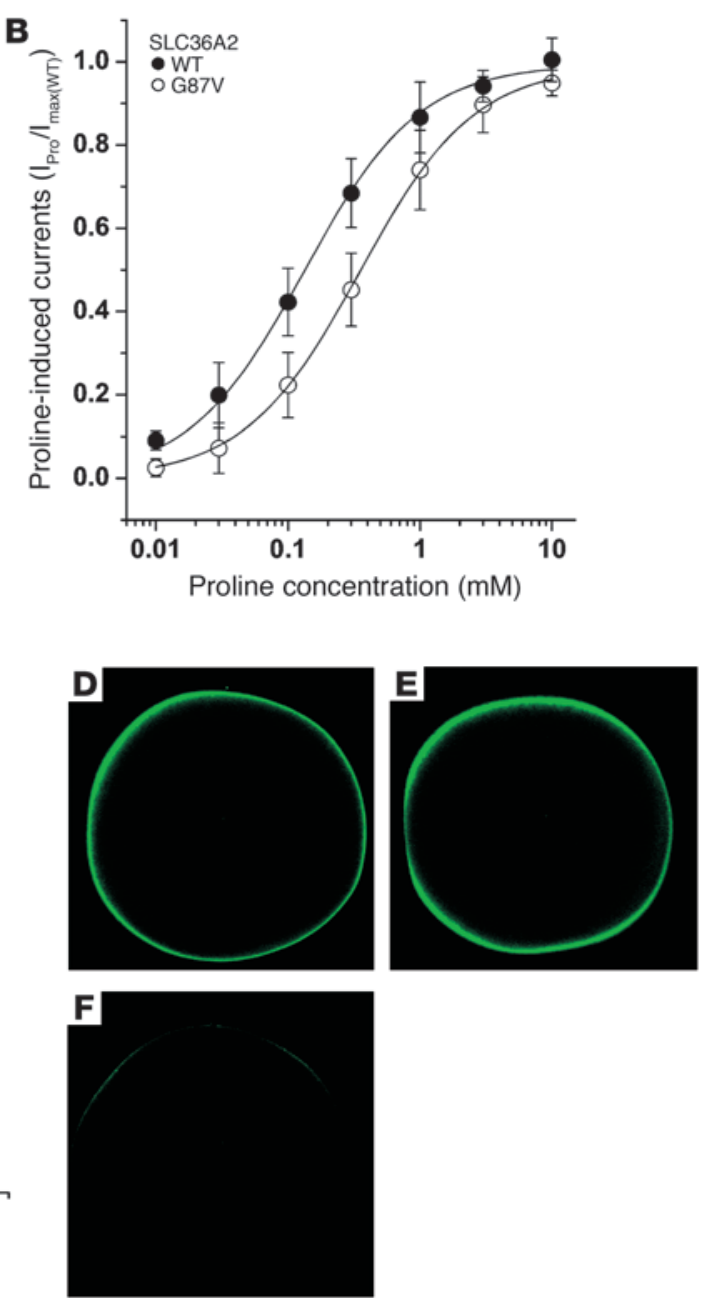

\section{Figure 4}

Transport activity of mutant proline and glycine transporters. Oocytes were injected with WT or mutant cRNA encoding the proton amino acid transporter $S \angle C 36 A 2$, the imino acid transporter SLC6A20, or the putative glycine transporter SLC6A18. (A) After incubation for 3 days (SLC36A2 WT and its mutant G87V) or 4 days (SLC6A18 and SLC6A2O WT and its mutant T199M), uptake of glycine and proline was measured. Each bar represents the mean \pm SD uptake activity of 10 oocytes. The experiment was repeated 3 times, with equivalent results. (B) Concentration dependence of proline-induced inward currents $\left(I_{\text {pro }}\right)$ induced in SLC36A2-expressing oocytes. Currents mediated by the G87V mutant are shown relative to the currents induced in WT transporter-expressing oocytes. Each data point represents the mean $\pm S D$ transport activity of $n=7$ oocytes. (C) Concentration dependence of glycine-induced inward currents in SLC36A2-expressing oocytes $(n=7$ experiments). Surface expression of eGFP-transporter fusions of WT (D) and G87V mutant (E) SLC36A2 in Xenopus laevis oocytes in comparison to noninjected oocytes (F). hypertension observed in the SLC6A18 nullizygous mouse model (26), the presence of homozygous stop codons in SLC6A18 in subject 5.2 did not result in HG in the absence of other mutations. This result together with the high frequency of the Y319X allele in the normal population suggests a minor role for this transporter in glycine transport in the human kidney. Genotype/phenotype comparison in family 7 , however, suggested that the SLC6A18 Y319X (and G496R) alleles are associated with increased urinary glycine levels (Table 1 and Supplemental Table 1). Our results are consistent with physiological data suggesting the presence of 4 different transporters for proline and glycine in the kidney, namely the common transporter SLC36A2, the specific proline transporter SLC6A20, the specific glycine transporter SLC6A18, and the general neutral amino acid transporter SLC6A19, which is affected in Hartnup disorder (20). At this stage, we cannot exclude the possibility that SLC6A18 is a nonfunctional transporter in humans and that another glycine-specific transporter might harbor mutations in IG or HG. However, because SLC36A2 is the major allele affected in the 2 disorders, the role of such a putative transporter would be a modifying one.

Neonatal screening studies report the frequency of IG to be around 1:10,000 $(11,41,43-45)$, with one exception reporting a higher frequency of 1:1,200 (11). An accurate estimate of IG frequency in adult populations has not been made, but approximately
$40 \%$ of neonatal IG disappears over the first year of life (11). This suggests an adult frequency of about 1:20,000, similar to that of other rare disorders. The SLC36A2 G87V allele was detected in all 3 French-Canadian pedigrees (nos. 1-3) and Australian pedigrees (nos. 4 and 5). The observed frequency of the SLC36A2 G87V allele, which did not cause IG in homozygotes, was 0.012 . Under random mating, it is expected to occur in the homozygous state at a frequency of $0.00014(1: 6,900)$. This allele only caused IG together with haploinsufficiency of the SLC6A20 T199M allele present mainly in European populations (frequency, 0.075). This variant of IG would thus occur with a combined frequency of $1.08 \times 10^{-5}$ $(1: 92,600)$. The SLC36A2 IVS1+1G $\rightarrow$ A allele occurred at a frequency of 0.004 . Homozygosity for this rare allele would be expected at a frequency of $1.6 \times 10^{-5}(1: 62,500)$.

This study suggests a model of imino acid and glycine reabsorption in the kidney (Figure 8) that permits prediction of the phenotype based on the genotype (Figure 9). The transporter for glycine and imino acids SLC36A2 is located in the earliest segment of the proximal tubule, as would be predicted for a highcapacity transporter (Figure 8). In the S2 and S3 segments of the proximal tubule, the low-capacity specific transporters for imino acids (SLC6A20) and glycine (SLC6A18) extract the residual amino acids. A reiterative process was used to estimate the contribution of each transporter to proline and glycine uptake in the kidney 
A

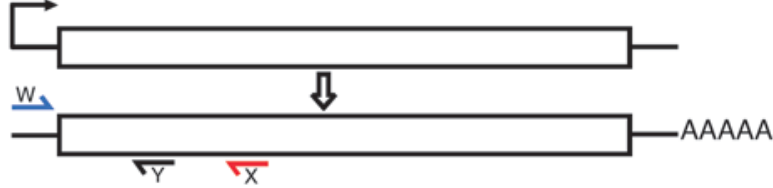

B

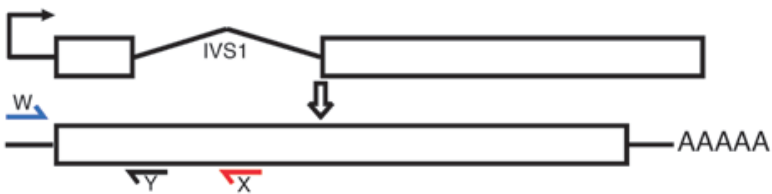

C
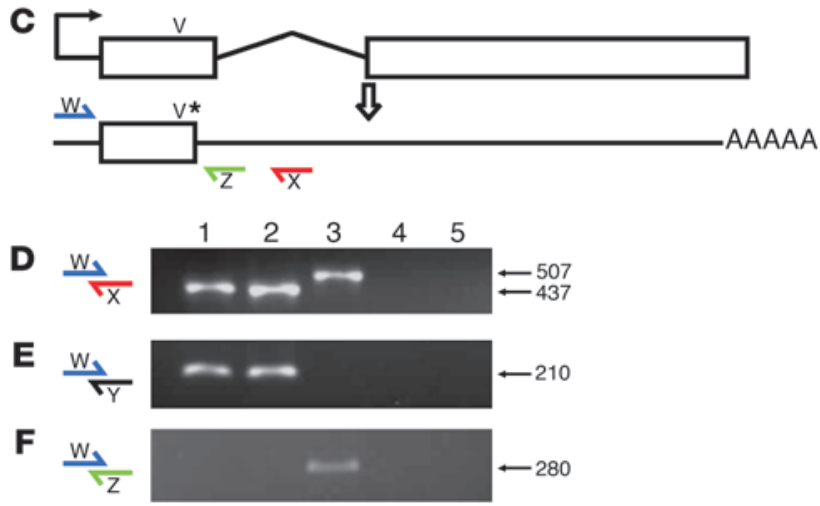

(Figure 9). The optimized model suggests that the human kidney has a higher capacity for proline uptake than for glycine uptake. Thus, mutations affecting iminoglycine transporters will more readily cause glycinuria than prolinuria. If it is assumed that $100 \%$ reabsorption capacity is required to remove all proline and glycine, the model predicts a total capacity of $140 \%$ for proline uptake, comprising SLC36A2 (60\%), SLC6A20 (40\%), and SLC6A19 (40\%). The total capacity for glycine transport would be $110 \%$, comprising SLC36A2 (40\%), SLC6A18 (10\%), and SLC6A19 (60\%). This model correctly replicates the phenotype in $95 \%$ of the cases in our cohort. Evidence has been presented that the paracellular pathway contributes to glycine reabsorption (46). The contribution of this pathway is difficult to estimate but could constitute part of the capacity that was attributed to SLC6A19.

The quantitative genotype/phenotype relationship of renal imino acid physiology (Figure 9) resolves a number of unexplained observations from previous cases. For example, pedigrees have been observed by our group (family 1) and Scriver and Hechtman (47), in which one parent with HG and the other with normal urine have a child with persistent IG. This can be explained by partially inactivating mutations not sufficient to cause HG alone and causing IG only in combination with more severe mutations or gene modifiers. In another well-recognized physiological variant, Greene et al. reported a case of IG with a normal maximal transport rate $\left(T_{m}\right)$ for proline but a marked splay in the renal tubular titration curve for proline reabsorption, consistent with a mutation affecting the $K_{m}$ of proline transport (14). This human physiological measurement accurately describes our functional analyses of the SLC36A2 G87V mutation (Figure 2), which has the same maximum velocity but a change of affinity for proline. Thus, it is proposed that phenotypes can be predicted from the genotype with high fidelity in this and other complex disorders when the contribution of each gene to the phenotype is known.

\section{Figure 5}

Analysis of the SLC36A2 IVS1 $+1 \mathrm{G} \rightarrow$ A splice donor site mutation using a minigene system. The mutation alters the consensus splice donor site (AClgtgagt). Each minigene contained an N-terminal c-myc epitope tag and a C-terminal FLAG tag for detection: (A) WT SLC36A2. (B) Minigene with 3,029-bp IVS1 containing the WT IVS1+1G allele. (C) Minigene with IVS1 containing the mutant IVS1+1A allele. The full-length cDNA is represented as the upper form, and the predicted expressed polyadenylated transcript as the lower form; $V$ indicates the location of the IVS1+1G $\rightarrow$ A mutation; the asterisk indicates the premature stop codon TGA. The SLC36A2 minigenes or a control vector with c-myc-tagged SLC6A20 were transfected into MDCKII cells and selected in G418 antibiotic, and RNA was extracted. The results of seminested RT-PCR using $5^{\prime}$ primer $W$ and different $3^{\prime}$ primers $\mathrm{X}, \mathrm{Y}$, or $\mathrm{Z}$ designed to qualitatively assess the effect of the IVS1+1G $\rightarrow$ A mutation on splicing: (D) WX, $X$ is within exon 2, (E) WY, $Y$ bridges the WT exon 1/exon 2 junction, (F) WZ, $Z$ bridges the mutant exon 1/exon 2 junction. Expected product sizes in bp are indicated. Lane 1, SLC36A2 full-length; lane 2, SLC36A2 WT IVS1+1G; lane 3, SLC36A2 mutant IVS1+1A; lane 4, SLC6A20 control; lane 5, first-round seminested negative control. All products were sequenced, and the results confirmed bioinformatic predictions that the $1 \mathrm{G} \rightarrow A$ mutation abrogated normal splicing of IVS1, leading to the use of an alternative splice donor site (AGlgtgggt) $70 \mathrm{bp}$ downstream. A premature stop codon within the alternative spliced transcript would truncate the protein at 55 amino acid residues.

In conclusion, the inactive or reduced function of SLC $36 \mathrm{~A} 2$ is the predominant determinant of the IG and HG phenotypes. Inactivating mutations in SLC6A20 and possibly SLC6A18 (or SLC6A19) account for additional features including the degree of urinary solute loss. Our model explains these related phenotypes by integrating observations from neonatal screening, developmental changes, and genetic and biochemical analyses of amino acid transporter genes and proteins. To our knowledge, this is the first explanation of an inherited disorder in terms of a major gene with its accompanying modifier genes for the reduced penetrance observed.

\section{Methods}

Institutional ethics committee approval was obtained for all studies in humans (University of Sherbrooke Health Centre 05-005 and 05-005R, Australian National University 2005/319, and Sydney South West Area Health Service X06-0172). Informed written consent was received from the subjects.

Collection and analysis of urine. Morning urine samples from subjects were tested at the Quebec Mass Urinary Screening Programme for hereditary metabolic disorders. Quantitative urinary amino acid analysis was performed by ion exchange chromatography using the Biochrom 20 or 30 platform (Biochrom Labs Inc.) (33). Sulfonated cation resin was used with lithium citrate buffers, and norleucine was included as an internal standard. All amino acids were measured using ninhydrin detection at an absorbance of $570 \mathrm{~nm}$, except for proline and hydroxyproline, measured at $440 \mathrm{~nm}$. Similar methods and chemistry were used to quantitate urinary amino acids from the Australian pedigrees.

Sequencing of candidate genes. DNA was isolated from 4-ml saliva samples collected using the Oragene DNA Self-Collection Kit (DNA Genotek). The genomic structures of SLC6A18, SLC6A19, SLC6A20, SLC36A1, and $S L C 36 A 2$ were determined, and primers were designed across the exonintron boundaries using Primer3 software (http://primer3.sourceforge. net) (Supplemental Table 2). PCR products were sequenced in forward and reverse directions. All chromatograms were viewed and contigs created 
A

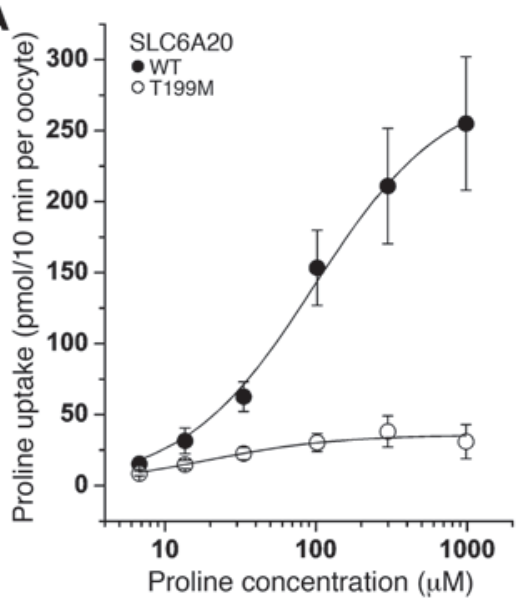

C

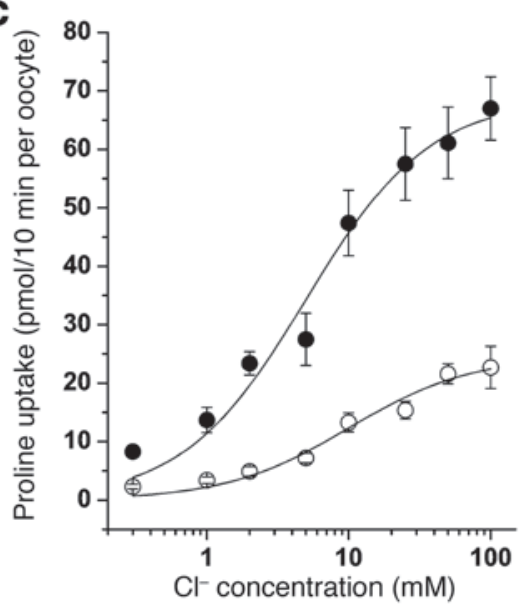

B

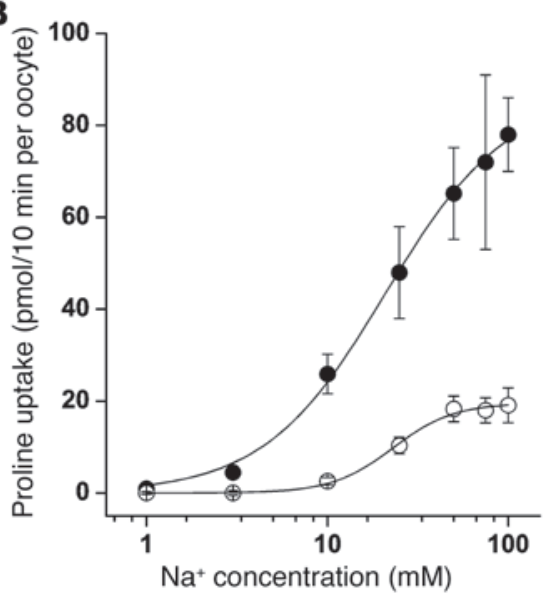

D
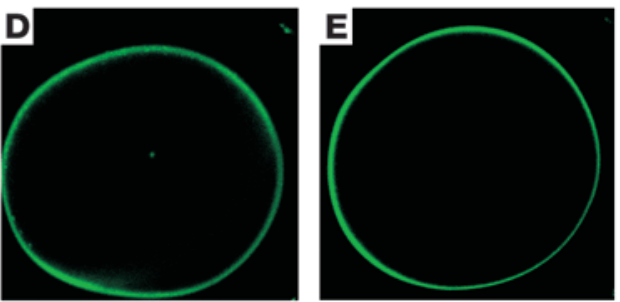

\section{Figure 6}

Transport activity of mutant SLC6A20. Oocytes were injected with WT or mutant cRNA encoding the imino acid transporter SLC6A20 (25 ng). After incubation for 4 days, $\left[{ }^{14} \mathrm{C}\right]$ proline uptake was determined in the presence of different concentrations of proline (A) and at a proline concentration of $50 \mu \mathrm{M}$ in the presence of different $\mathrm{Na}^{+}$concentrations (B) or different concentrations of $\mathrm{Cl}^{-}(\mathbf{C})$. Each data point represents the mean \pm SD transport activity of $n=10$ oocytes. Surface expression of eGFP-transporter fusions of WT (D) and mutant T199M (E) SLC6A20 in Xenopus laevis oocytes in comparison to noninjected oocytes (F). Each experiment was repeated 3 times, with equivalent results. using Sequencher 4.8 (Gene Codes Corp.). Orthologs of human SLC36A2, SLC6A20, and SLC6A18 proteins and other members in the amino acid transporter branch (II) of the SLC6 family were obtained from Ensembl release 47 (www.ensembl.org). All sequence alignments were created using the ClustalW algorithm in MacVector 9.5 (MacVector Inc.).
Allele frequencies. A panel of 590 normal Australian genomic DNAs and an additional panel of 95 genomic DNA samples from French-Canadian subjects (obtained from the University of Montreal, Montreal, Quebec, Canada) were screened to determine the allele frequency of novel mutations. SLC36A2 G87V was determined by an RFLP resulting from inac-
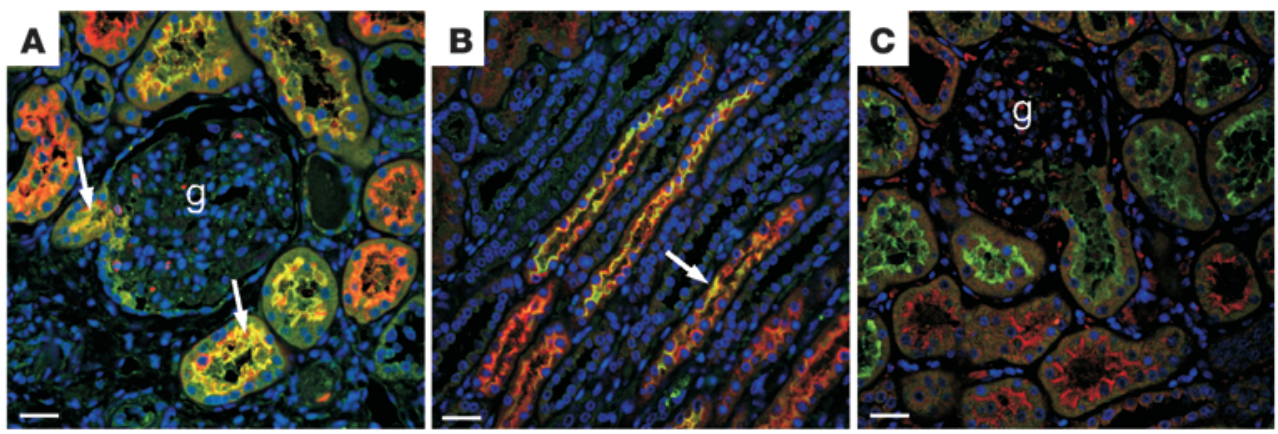

\section{Figure 7}

Expression of SLC36A2 and SLC6A20 in adult human kidney. (A) Immunofluorescence of SLC36A2 (green) revealed colocalization (yellow) with the proximal tubule marker Lotus teragonolobus agglutinin (LTA; red) on the apical membrane of the S1 segment of proximal tubules. Arrows indicate SLC36A2 expression in the S1 segment of a proximal tubule close to the glomerulus (g). (B) Immunofluorescence of SLC6A20 (green) revealed colocalization (yellow) with LTA (red) on the apical membrane of the S2-S3 segment of proximal tubules. Arrow indicates SLC6A20 expression in the S3 segment of a proximal tubule. (C) Colocalization was not observed in sections stained for both SLC36A2 (green) and SLC6A20 (red). Negative controls for A, B, and C showed no staining (data not shown). Original magnification, $\times 40$. Scale bars: $30 \mu \mathrm{m}$. 


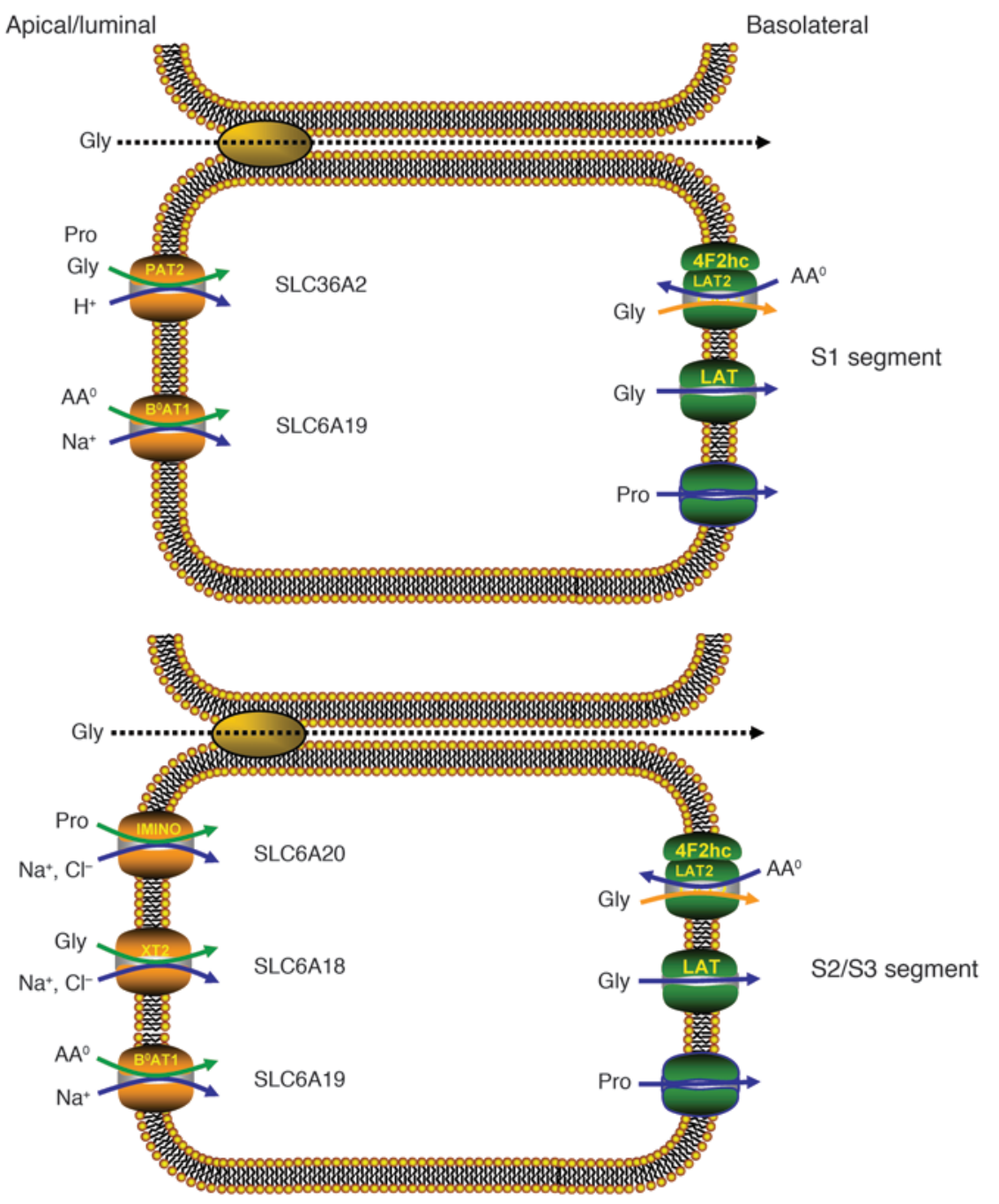

Figure 8

Model of imino acid and glycine reabsorption in the kidney. Different transporters in the $\mathrm{S} 1$ and $\mathrm{S} 2 / \mathrm{S} 3$ segments of the proximal tubule mediate uptake of imino acids and glycine. In the S1 segment, the main transporters are SLC36A2 and SLC6A19, which both transport imino acids and glycine. In the S2/S3 segment, imino acid transport is mediated by SLC6A20, whereas glycine transport is mediated by SLC6A18. SLC6A19 contributes to neutral amino acid $\left(A A^{0}\right)$ reabsorption throughout the proximal tubule. Paracellular transport is an alternate mechanism of glycine reabsorption (dashed arrow). tivation of an ApaI restriction enzyme site and SLC36A2 IVS1+1G $\rightarrow$ A was determined by an RFLP resulting from inactivation of an $\mathrm{HpyCH} 4 \mathrm{IV}$ restriction enzyme site. A custom Taqman SNP assay (Applied Biosystems) run on a Rotor-Gene RG3000 (Corbett Life Sciences) was used to measure the frequency of SLC6A18 G496R. Allele frequencies of known SNPs SLC6A18 Y319X, SLC6A20 T199M, and SLC6A19 IVS7-4G $\rightarrow$ A were confirmed by allele-specific PCR. Primers, products, and probes are detailed in Supplemental Table 3.

Molecular cloning and site-directed mutagenesis. Human SLC36A2, SLC6A20, and SLC6A18 were amplified by RT-PCR from human kidney RNA (BD Biosciences). For mutant transporter studies, pGEM-He-Juel (48) plasmids containing SLC36A2, SLC6A20, or SLC6A18 transporters were used as templates for site-directed mutagenesis using the QuikChange II Kit (Stratagene). All introduced mutations were confirmed by sequencing. Primers and products are detailed in Supplemental Table 3.

Minigene construction. The cDNAs for SLC36A2 and SLC6A20 were PCR amplified from human kidney cDNA (BD Biosciences) using DyNAzyme EXT (New England BioLabs Inc.) and cloned into pGEM-T-Easy (Promega). A 326-bp fragment containing the ATG start codon and exons 1 and 2 of SLC36A2 was PCR amplified with MluI ends from pGEM-TSLC36A2 and cloned into pEF-BOS for addition of an N-terminal c-myc tag (Supplemental Table 2). Genomic DNA from subjects 7.3 (normal) and 7.6 (IG) were used as templates for amplification of $3.3-\mathrm{kb}$ fragments con- taining exon 1, the entire first intron with WT IVS $+1 \mathrm{G}$ or mutant IVS+1A alleles, and part of exon 2. These were ligated into pGEM-T-Easy and then subcloned into pEF-BOS with MluI overhangs. A 1,247-bp BamHI/ XhoI-ended PCR product containing SLC36A2 exons 2-10 and with the stop codon removed was cloned into pcDNA3.1 (Invitrogen) adapted for C-terminal FLAG epitope tagging. Full-length, WT, and mutant SLC36A2 minigenes were constructed by subcloning EcoRI/BamHI fragment 233, 3,321 , or $3321 \mathrm{bp}$ from pEF-BOS plasmids into pcDNA3.1-SLC36A2FLAG. Each minigene had an $\mathrm{N}$-terminal c-myc tag and a C-terminal FLAG tag. SLC6A20 cDNA was cloned into pcDNA3.1 containing a c-myc epitope as a negative control for minigene expression.

Cell culture and immunofluorescence. MDCKII cells were maintained in DMEM supplemented with $10 \%$ (v/v) FCS, penicillin, and streptomycin. MDCKII cells were transfected with $2 \mu \mathrm{g}$ pcDNA3.1 vectors containing SLC36A2 minigenes using $10 \mu \mathrm{l}$ Lipofectamine 2000 (Invitrogen). After 48 hours, $500 \mu \mathrm{g} / \mathrm{ml} \mathrm{G} 418$ was added for a period of 10 days. G418-resistant pools were transferred to 8 -well chamber slides (BD Biosciences) coated in $5 \mu \mathrm{g} / \mathrm{ml}$ poly-L-lysine for 30 minutes. The cells were cultured for 3 more days, washed once in PBS, fixed in 50\% methanol $/ 50 \%$ ethanol $(\mathrm{v} / \mathrm{v})$ for 10 minutes at $-30^{\circ} \mathrm{C}$, air-dried, and rehydrated in PBS. After blocking for 1 hour at $37^{\circ} \mathrm{C}$ in PBS containing 20\% (v/v) BlokHen II (AvesLab) and $0.1 \%(\mathrm{v} / \mathrm{v})$ Triton X-100, mouse monoclonal anti-c-myc (clone 4A6; Upstate) or anti-FLAG (clone M2; Sigma-Aldrich) was added 


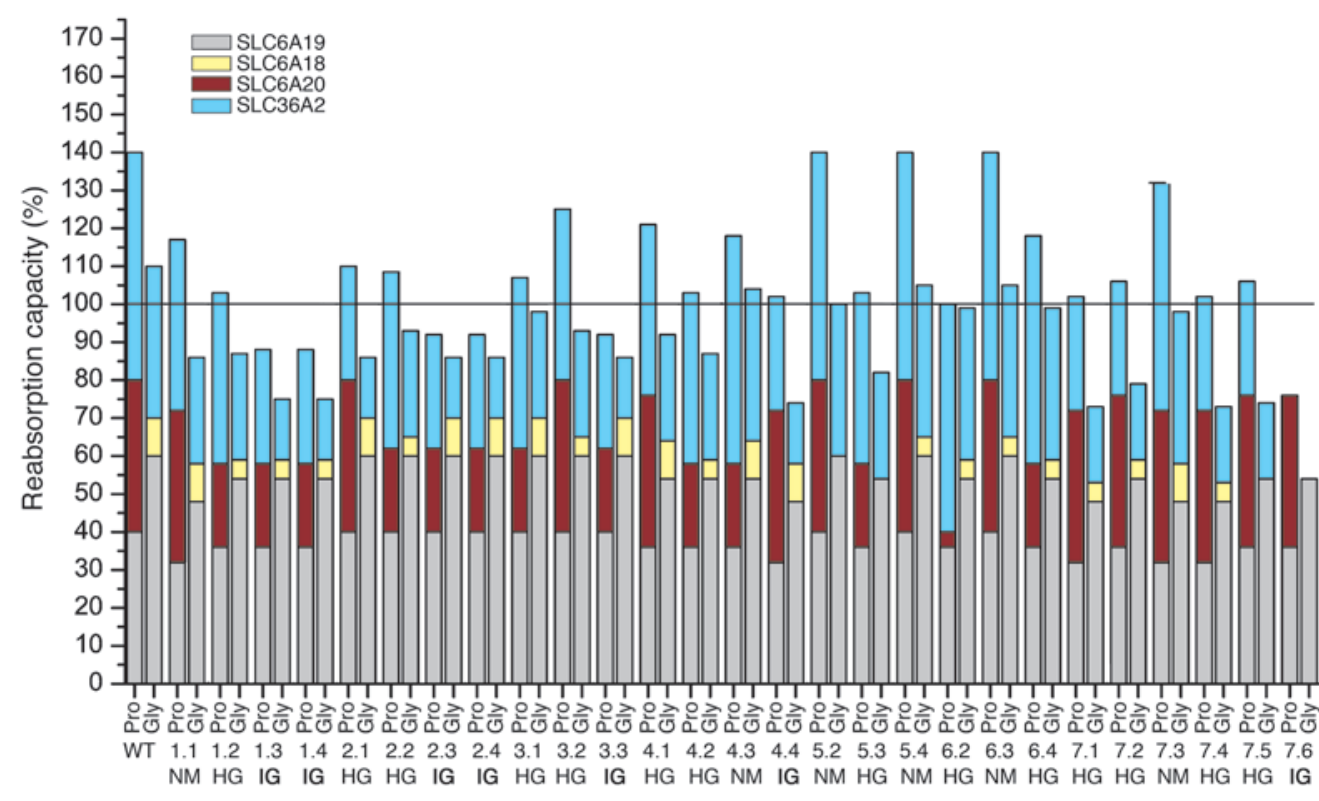

Figure 9

Prediction of the renal phenotype from the genotype. The results of in vivo urine analysis and in vitro transport experiments were combined to estimate the contribution of SLC6A19, SLC6A18, SLC6A20, and SLC36A2 to proline and glycine reabsorption in the kidney. Reabsorption capacity above $100 \%$ would manifest as a normal urinary phenotype. The urine phenotype is indicated below the subject as normal (NM), HG, or IG. The following formula was used to estimate proline and glycine reabsorption: (activity of allele $1+$ activity of allele 2) $/ 2 \times$ contribution of SLC36A2 + (activity of allele $1+$ activity of allele 2 ) $/ 2 \times$ contribution of SLC6A18 + (activity of allele $1+$ activity of allele 2 ) $/ 2 \times$ contribution of SLC6A19 + (activity of allele $1+$ activity of allele 2)/ $2 \times$ contribution of SLC6A20. The following contributions to proline transport were used: SLC36A2 (60 AU); SLC6A18 (0 AU, not a substrate); SLC6A19 (40 AU); SLC6A20 (40 AU). The following contributions to glycine transport were used: SLC36A2 (40 AU); SLC6A18 (10 AU); SLC6A19 (60 AU); SLC6A20 (0 AU, not a substrate). The activity of individual alleles was calculated as a fraction of 1 , reflecting the activity of the mutant compared with the WT when expressed in Xenopus laevis oocytes. The algorithm correctly replicates the urinary phenotype of 25 of the 28 individuals.

in PBS/BlokHen at 1:1,000 dilution for 1 hour at room temperature. Anti-mouse $\mathrm{F}\left(\mathrm{ab}^{\prime}\right)_{2}$ conjugated with Alexa Fluor 594 (Invitrogen) diluted 1:500 in PBS/BlokHen was added for 1 hour at room temperature. In the final wash step, $1 \mu \mathrm{g} / \mathrm{ml}$ DAPI was added, and the slides were mounted in ProLong Gold antifade reagent (Invitrogen).

RT-PCR for splice site inactivation. G418-resistant MDCKII cells transfected with SLC36A2 minigenes were grown in 6-well plates for 48 hours. Total RNA was isolated using TRIzOL (Invitrogen) for seminested PCR with $5^{\prime}$ primer $\mathrm{W}$ and $3^{\prime}$ primers $\mathrm{X}, \mathrm{Y}$, or $\mathrm{Z}$. Primer $\mathrm{W}$ binds within the c-myc epitope, primer $X$ binds within exon 4, primer $Y$ spans the exon 1-exon 2 boundary, and primer $Z$ binds the alternate exon 1/exon 2 boundary. Primers and products are detailed in Supplemental Table 3.

Tissue staining. De-identified normal human adult kidney specimens were obtained with ethics approval (Sydney South West Area Health Service, Ethics Review Committee [RPAH Zone], X04-0239). Tissues were fixed in a 20 -fold excess volume of $10 \%(\mathrm{v} / \mathrm{v})$ neutral buffered formalin for at least 24 hours. Deparaffinized slides were subjected to citrate buffer antigen retrieval before application of $200 \mu \mathrm{l}$ of PBS/BlokHen to each section. Primary antibodies used were: polyclonal rabbit anti-human SLC36A2 (raised against peptide comprising amino acids 25-45 of human SLC36A2; Pineda Antikörper-Service), diluted 1:600, and mouse monoclonal antihuman SLC6A20 (clone 3G6; Abnova), diluted 1:200. Secondary antibodies included goat anti-rabbit Alexa Fluor 488 (1:200; Invitrogen) and antimouse $\mathrm{F}\left(\mathrm{ab}^{\prime}\right)_{2}$ Alexa Fluor 594 (1:500; Invitrogen). Proximal tubules were identified using a biotinylated lectin marker, Lotus tetragonolobus agglutinin (LTA; 1:200; Vector Laboratories), and detected with streptavidin-Alexa Fluor 594 (Invitrogen). All antibodies were diluted in PBS containing $0.04 \%$ (v/v) Triton X-100. Digital immunofluorescence images were obtained using a $\times 40$ air or $\times 60$ water objective with a Nikon C1 confocal system installed on a Nikon TE2000-E microscope, using the 405-nm, 488-nm, and 561-nm lasers. Images were equally processed using Adobe Photoshop 7.0.

Oocyte flux and electrophysiology studies. Oocyte isolation and maintenance have been described in detail (49). For expression studies, the pGEM-He-Juel plasmids containing SLC6A18, SLC6A20, and SLC36A2 were linearized with NotI or SalI and transcribed in vitro using the $\mathrm{T} 7 \mathrm{mMessage}$ mMachine Kit (Ambion; Applied Biosystems.). Oocytes were each injected with 25 ng of complementary RNA (cRNA) encoding human SLC6A18 or SLC6A20 or $5 \mathrm{ng}$ of SLC36A2. Uptake of glycine $(100 \mu \mathrm{M}$ for SLC36A2 and $10 \mu \mathrm{M}$ for SLC6A18) and proline $(100 \mu \mathrm{M})$ was determined using an incubation period of 10-20 minutes. Subsequently, oocytes were dissolved, and the amount of $\left[{ }^{14} \mathrm{C}\right]$ proline or $\left[{ }^{14} \mathrm{C}\right]$ glycine was determined by scintillation counting. To determine $\mathrm{Na}^{+}$dependence, $\mathrm{NaCl}$ was replaced by $\mathrm{N}$-methyl-D-glucamine chloride (NMDG-Cl); to determine $\mathrm{Cl}^{-}$dependence, $\mathrm{NaCl}$ was replaced by Na gluconate in the transport buffer.

Amino acid-induced currents were analyzed by 2 -electrode voltage clamp recording (all equipment from Axon Instruments). Oocytes that had a membrane potential of less than $-30 \mathrm{mV}$ were chosen. Once a stable membrane potential was reached under current clamp conditions, the amplifier was switched to voltage clamp mode to maintain $-50 \mathrm{mV}$. Oocytes were superfused with ND96 buffer $\left(96 \mathrm{mM} \mathrm{NaCl}, 2 \mathrm{mM} \mathrm{KCl}, 1.8 \mathrm{mM} \mathrm{CaCl}_{2}\right.$, $1 \mathrm{mM} \mathrm{MgCl}_{2}, 5 \mathrm{mM}$ HEPES, $\mathrm{pH}$ 7.4) containing substrates as indicated. The current generated by the transporter at a given membrane potential was calculated as the difference of the currents measured in the presence and the absence of substrate.

Construction of fusion proteins and surface expression. The cDNAs of SLC6A18, SLC6A20, and SLC36A2 were excised with EcoRI and subcloned into pEGFP-C3 (BD Biosciences). In vitro transcription of the eGFP-transporter fusion was initiated from the $\mathrm{T} 7$ promoter incorporated into the 5' primer sequence (see Supplemental Table 3). The cRNA was extended by polyadenylation using a Poly(A) Tailing Kit (Ambion). After purification, 25 ng cRNA was injected into oocytes, and surface expression was analyzed after 5 days incubation by confocal microscopy using a Bio-Rad Radiance 2000 inverted confocal microscope. We have previously shown 
that the laser light does not penetrate far into the oocyte because of its egg yolk content. As a result, membrane proteins in the endoplasmic reticulum or Golgi apparatus are not visible; only proteins expressed at the surface are excited (50). The fluorescence intensity is therefore proportional to the surface expression.

Transporter model construction. A topology plot was generated for SLC36A2 using transmembrane helix prediction as calculated by the TMHMM server (http://www.cbs.dtu.dk/services/TMHMM/). The model was confirmed using the TOPCONS website (http://topcons.cbr.su.se/). Homology models of SLC6A18 and SLC6A20 were derived from the bacterial homolog of the $\mathrm{Na}^{+} / \mathrm{Cl}^{-}$-dependent neurotransmitter LeuT $\mathrm{T}_{\mathrm{Aa}}(51)$. Optimally aligned sequences were submitted to the Swiss-PDB server (52), and the structures generated were visualized using the PyMol program (DeLano Scientific).

\section{Acknowledgments}

We thank the families who consented to participate in this study. Financial support was provided by the Australian National Health and Medical Research Council (project grant 402730), the Australian Research Council (Discovery Project DP0877897), the Private Practice Fund at The Canberra Hospital, the Rebecca L. Cooper Foundation, and The Cell and Gene Trust. Assistance in clinical amino acid quantitation was provided by Robert Giguère of the Biochemical Genetics Service, University of Sherbrooke Health Centre, and Kevin Carpenter of the NSW Biochemical Genetics and Newborn Screening Services at The Children's Hospital at Westmead. The identification of individuals with neonatal IG would not have been possible without the pioneering studies of Bridget Wilcken. We thank Robyn Soper of the Department of Anatomical Pathology, Royal Prince Alfred Hospital, for assistance; and Jean-Baptiste Rivière and Guy Rouleau of the Centre of Excellence in Neuromics, University of Montreal, for normal French-Canadian human genomic DNA samples.

Received for publication June 30, 2008, and accepted in revised form October 1, 2008.

Address correspondence to: John E.J. Rasko, Centenary Institute, Locked Bag No. 6, Newtown 2042, New South Wales, Australia. Phone: 61-2-95656156; Fax: 61-2-95656101; E-mail: j.rasko@centenary.usyd.edu.au.

Stefan Bröer and Charles G. Bailey contributed equally to this work.
1. Baron, D.N., Dent, C.E., Harris, H., Hart, E.W., and Jepson, J.B. 1956. Hereditary pellagra-like skin rash with temporary cerebellar ataxia, constant renal amino-aciduria, and other bizarre biochemical features. Lancet. 271:421-428.

2. Penrose, L.S. 1935. Inheritance of phenylpyruvic amentia (phenylketonuria). Lancet. 2:192-194.

3. Debre, R., et al. 1958. Genetics of haemochromatosis. Ann. Hum. Genet. 23:16-30.

4. Chesney, R.W. 2001. Iminoglycinuria. In The metabolic and molecular bases of inherited diseases. C.R. Scriver, A.L. Beaudet, W.S. Sly, and D. Valle, editors. McGrawHill. New York, New York, USA. 4971-4982.

5. Joseph, R., Ribierre, M., Job, J.C., and Girault, M. 1958. Familial disease with associated convulsions with very early onset, excess albumin in the cerebrospinal fluid \& hyperaminoaciduria [In French]. Arch. Fr. Pediatr. 15:374-387.

6. Scriver, C.R., Schafer, I.A., and Efron, M.L. 1961. New renal tubular amino-acid transport system and a new hereditary disorder of amino-acid metabolism. Nature. 192:672-673.

7. Whelan, D.T., and Scriver, C.R. 1968. Cystathioninuria and renal iminoglycinuria in a pedigree. N. Engl. J. Med. 278:924-927.

8. Rosenberg, L.E., Durant, J.L., and Elsas, L.J. 1968. Familial iminoglycinuria. An inborn error of renal tubular transport. N. Engl. J. Med. 278:1407-1413.

9. Scriver, C.R., and Wilson, O.H. 1967. Amino acid transport: evidence for genetic control of two types in human kidney. Science. 155:1428-1430.

10. Scriver, C.R. 1968. Renal tubular transport of proline, hydroxyproline, and glycine. 3. Genetic basis for more than one mode of transport in human kidney. J. Clin. Invest. 47:823-835.

11. Procopis, P.G., and Turner, B. 1971. Iminoaciduria: a benign renal tubular defect. J. Pediatr. 79:419-422.

12. Kaser, H., Cottier, P., and Antener, I. 1962. Glucoglycinuria, a new familial syndrome. J. Pediatr. 61:386-394.

13. De Vries, A., Kochwa, S., Lazebnik, J., Frank, M., and Djaldetti, M. 1957. Glycinuria, a hereditary disorder associated with nephrolithiasis. Am. J. Med. 23:408-415.

14. Greene, M.L., Lietman, P.S., Rosenberg, L.E., and Seegmiller, J.E. 1973. Familial hyperglycinuria. New defect in renal tubular transport of glycine and imino acids. Am. J. Med. 54:265-271.

15. Statter, M., Ben-Zvi, A., Shina, A., Schein, R., and Russell, A. 1976. Familial iminoglycinuria with normal intestinal absorption of glycine and imino acids in association with profound mental retardation, a possible "cerebral phenotype". Helv. Paediatr. Acta. 31:173-182.

16. Saito, T., et al. 1981. Atypical gyrate atrophy of the choroid and retina and iminoglycinuria. TohokuJ. Exp. Med. 135:331-332.

17. Tancredi, F., Guazzi, G., and Auricchio, S. 1970. Renal iminoglycinuria without intestinal malabsorption of glycine and imino acids. J. Pediatr. 76:386-392.

18. Fraser, G.R. 1971. More on renal iminoglycinuria. J. Pediatr. 79:174.

19. Lasley, L., and Scriver, C.R. 1979. Ontogeny of amino acid reabsorption in human kidney. Evidence from the homozygous infant with familial renal iminoglycinuria for multiple proline and glycine systems. Pediatr. Res. 13:65-70.

20. Bröer, S. 2008. Amino acid transport across mammalian intestinal and renal epithelia. Physiol. Rev. 88:249-286.

21. Boll, M., Daniel, H., and Gasnier, B. 2004. The SLC36 family: proton-coupled transporters for the absorption of selected amino acids from extracellular and intracellular proteolysis. Pflugers Arch. 447:776-779.

22. Anderson, C.M., et al. 2004. H+/amino acid transporter 1 (PAT1) is the imino acid carrier: an intestinal nutrient/drug transporter in human and rat. Gastroenterology. 127:1410-1422.

23. Camargo, S.M., Bockenhauer, D., and Kleta, R. 2008. Aminoacidurias: clinical and molecular aspects. Kidney Int. 73:918-925.

24. Kowalczuk, S., et al. 2005. Molecular cloning of the mouse IMINO system: an $\mathrm{Na}^{+-}$and $\mathrm{Cl}^{-}$-dependent proline transporter. Biochem. J. 386:417-422.

25. Takanaga, H., Mackenzie, B., Suzuki, Y., and Hediger, M.A. 2005. Identification of mammalian proline transporter SIT1 (SLC6A20) with characteristics of classical system imino. J. Biol. Chem. 280:8974-8984.

26. Quan, H., et al. 2004. Hypertension and impaired glycine handling in mice lacking the orphan transporter XT2. Mol. Cell. Biol. 24:4166-4173.

27. Nash, S.R., et al. 1998. Cloning, gene structure and genomic localization of an orphan transporter from mouse kidney with six alternatively-spliced isoforms. Receptors Channels. 6:113-128.

28. Seow, H.F., et al. 2004. Hartnup disorder is caused by mutations in the gene encoding the neutral amino acid transporter SLC6A19. Nat. Genet.
36:1003-1007.

29. Kleta, R., et al. 2004. Mutations in SLC6A19, encoding B0AT1, cause Hartnup disorder. Nat. Genet. 36:999-1002.

30. Azmanov, D.N., et al. 2007. Persistence of the common Hartnup disease D173N allele in populations of European origin. Ann. Hum. Genet. 71:755-761.

31. Silbernagl, S. 1988. The renal handling of amino acids and oligopeptides. Physiol. Rev. 68:911-1007.

32. Böhmer, C., et al. 2005. Characterization of mouse amino acid transporter B0AT1 (slc6a19). Biochem. J. 389:745-751.

33. Wilcken, B., Wiley, V., Hammond, J., and Carpenter, K. 2003. Screening newborns for inborn errors of metabolism by tandem mass spectrometry. N. Engl. J. Med. 348:2304-2312.

34. Auray-Blais, C., Cyr, D., and Drouin, R. 2007. Quebec neonatal mass urinary screening programme: from micromolecules to macromolecules. J. Inherit. Metab. Dis. 30:515-521.

35. Nalla, V.K., and Rogan, P.K. 2005. Automated splicing mutation analysis by information theory. Hum. Mutat. 25:334-342.

36. Smith, P.J., et al. 2006. An increased specificity score matrix for the prediction of SF2/ASF-specific exonic splicing enhancers. Hum. Mol. Genet. 15:2490-2508.

37. Eslami, B., et al. 2006. A nonsense polymorphism (Y319X) of the solute carrier family 6 member 18 (SLC6A18) gene is not associated with hypertension and blood pressure in Japanese. Tohoku J. Exp. Med. 208:25-31.

38. Höglund, P.J., Adzic, D., Scicluna, S.J., Lindblom, J., and Fredriksson, R. 2005. The repertoire of solute carriers of family 6 : identification of new human and rodent genes. Biochem. Biophys. Res. Commun. 336:175-189.

39. Bröer, S. 2006. The SLC6 orphans are forming a family of amino acid transporters. Neurochem. Int. 48:559-567.

40. Brodehl, J., and Gellissen, K. 1968. Endogenous renal transport of free amino acids in infancy and childhood. Pediatrics. 42:395-404.

41. Scriver, C., and Tenenhouse, H.S. 1992. Mendelian phenotypes as 'probes' of renal transport systems for amino acids and phosphate. In Handbook of physiology. E.E. Windhager, editor. Oxford University Press. Oxford, United Kingdom. 1977-2016.

42. Scriver, C.R., Arthus, M.F., and Bergeron, M. 1982. Neonatal iminoglycinuria: evidence that the pro- 
linuria originates in selective deficiency of transport activity in the proximal nephron. Pediatr. Res. 16:684-687.

43. Wilcken, B., Smith, A., and Brown, D.A. 1980. Urine screening for aminoacidopathies: is it beneficial? Results of a long-term follow-up of cases detected by screening one million babies. J. Pediatr. 97:492-497.

44. Lemieux, B., Auray-Blais, C., Giguère, R., Shapcott, D., and Scriver, C.R. 1988. Newborn urine screening experience with over one million infants in the Quebec Network of Genetic Medicine. J. Inherit. Metab. Dis. 11:45-55.

45. Auray-Blais, C., Giguère, R., and Lemieux, B. 2003.
Newborn urine screening programme in the province of Quebec: an update of 30 years' experience. J. Inherit. Metab. Dis. 26:393-402.

46. Silbernagl, S., Foulkes, E.C., and Deetjen, P. 1975 Renal transport of amino acids. Rev. Physiol. Biochem. Pharmacol. 74:105-167.

47. Scriver, C.R., and Hechtman, P. 1970. Human genetics of membrane transport with emphasis on amino acids. Adv. Hum. Genet. 1:211-274.

48. Bröer, S., et al. 1999. Characterization of the highaffinity monocarboxylate transporter MCT2 in Xenopus laevis oocytes. Biochem. J. 341:529-535.

49. Bröer, S. 2003. Xenopus laevis Oocytes. Methods
Mol. Biol. 227:245-258.

50. Chubb, S., Kingsland, A.L., Bröer, A., and Bröer, S. 2006. Mutation of the 4F2 heavy-chain carboxy terminus causes $y^{+}$LAT2 light-chain dysfunction. Mol. Membr. Biol. 23:255-267.

51. Yamashita, A., Singh, S.K., Kawate, T., Jin, Y., and Gouaux, E. 2005. Crystal structure of a bacterial homologue of $\mathrm{Na}+/ \mathrm{Cl}$--dependent neurotransmitter transporters. Nature. 437:215-223.

52. Arnold, K., Bordoli, L., Kopp, J., and Schwede, T. 2006. The SWISS-MODEL workspace: a web-based environment for protein structure homology modelling. Bioinformatics. 22:195-201. 